\title{
Randomised, blinded, placebo controlled trial of doxycycline for chronic seronegative arthritis
}

\author{
M Smieja, D W MacPherson, W Kean, M L Schmuck, C H Goldsmith, W Buchanan, \\ L E Hart, J B Mahony
}

\begin{abstract}
Objective-To determine whether long term doxycycline improves symptoms in patients with chronic seronegative or reactive arthritis.

Methods-A randomised, triple blind, controlled clinical trial of three months' treatment with doxycycline or placebo of patients with chronic reactive or seronegative arthritis was conducted. The primary study end points were three month pain and functional status measured by a self administered Arthritis Impact Measurement Scales version 2 (AIMS2) quality of life questionnaire. Secondary end points were pain and functional status at 6-12 months, three month rheumatologist assessed joint count, pain, and arthritis activity, and treatment efficacy in those with previous exposure to chlamydia.

Results-Of 60 patients randomly allocated to receive doxycycline or placebo, results from 37 were evaluable at three months. Groups were well balanced for major prognostic variables. Doxycycline had no detectable effect at three months on pain change scores (mean difference $1.5,95 \% \mathrm{CI}$ -1.2 to $4.2, p=0.25$ ) or composite functional change scores (mean difference $0.8,95 \% \mathrm{CI}$ -5.6 to $7.1, p=0.81)$. Furthermore, there were no differences in secondary study end points, and no apparent treatment effect in patients with previous chlamydia infection. Conclusion-Three months' treatment with doxycycline did not improve pain or functional status in patients with chronic reactive or seronegative arthritis.

(Ann Rheum Dis 2001;60:1088-1094)
\end{abstract}

Department of Clinica Epidemiology and Biostatistics,

McMaster University

C H Goldsmith

Education Services, McMaster University M L Schmuck

Correspondence to: Dr M Smieja, Laboratory Medicine L424, St Joseph's Hospital, 50 Charlton Avenue East, Hamilton ON L8N 4A6, Canada

smiejam@mcmaster.ca

Accepted 8 June 2001 B27 positive) subjects, reactive arthritis is causally associated with a number of genitourinary or gastrointestinal Gram negative bacterial pathogens. ${ }^{12}$ Arthritis occurs in $1-15 \%$ of patients with inflammatory gastroenteritis owing to Salmonella, Shigella, Campylobacter, or Yersinia species, ${ }^{2-4}$ or after cervicitis or urethritis with Chlamydia trachomatis. ${ }^{5-8}$ Pharyngitis or dermatitis caused by Streptococcus pyogenes, and respiratory infection due to Chlamydia pneumoniae, may also cause arthritis, ${ }^{910}$ but streptococcal associated arthritis is often considered distinct from reactive arthritis, whereas a causal role for $C$ pneumoniae in arthritis remains unclear. Up to $50 \%$ of reactive arthritis, and up to $30 \%$ of undifferentiated seronegative arthritis, may be attributable to $C$ trachomatis infection. ${ }^{11}{ }^{12}$

In $20-50 \%$ of patients with acute reactive arthritis, chronic arthritis symptoms follow. ${ }^{1-4}$ In patients with post-salmonella reactive arthritis, acute symptoms settled by four months. ${ }^{4}$ In patients with chronic symptoms, a role for chronic or recurrent infection has been postulated, ${ }^{13-16}$ and bacterial DNA and antigen have been detected in synovial fluid. ${ }^{14}$ 17-20 If chronic infection has an aetiologic role in the maintenance of chronic arthritis, an appropriate antibiotic should decrease the inflammation and improve pain and joint function.

In randomised controlled trials, 5-14 days of antibiotic treatment were ineffective in reducing symptoms or disease activity in patients with reactive arthritis. ${ }^{2122}$ The single published randomised study of long term antibiotics available at the time that we designed our study indicated that three months of lymecycline (a drug related to tetracycline but not available in Canada) may induce symptom remission in chlamydia associated acute reactive arthritis. ${ }^{23}$

In this study we tested the hypotheses that three months' treatment with doxycycline improves symptoms of reactive arthritis in general, and of arthritis associated with chlamydia exposure in particular. As acute reactive arthritis is often self limited, ${ }^{3}$ we chose to study patients with chronic symptoms (six months or more). We further suggested that patients with undifferentiated seronegative arthritis represented a spectrum of reactive arthritis, ${ }^{1}$ and designed a "pragmatic" or "effectiveness" study with broad inclusion criteria to test whether long term antibiotics improve pain and functional status of patients with reactive or seronegative arthritis.

\section{Patients and methods}

PATIENTS

Consecutive patients referred by family doctors to a participating academic rheumatologist affiliated with McMaster University in Hamilton, Ontario, Canada, were invited to participate in this study. We enrolled consenting patients aged 18-65 years with oligoarthritis or 
polyarthritis, signs of acute joint inflammation on initial rheumatological examination, and with negative laboratory tests for rheumatoid factor and antinuclear antibodies. $x$ Ray examinations of sacroiliac joints and other laboratory tests were obtained according to the rheumatologist's usual practice. The requirement for six months of symptoms before enrolment was modified during the study, and six patients with symptoms for four or five months were also enrolled. Patients with psoriatic lesions were eligible if the skin manifestations appeared within one year of arthritis onset. Exclusions were a history of allergy or sensitivity to doxycycline or tetracycline; concurrent or recent use of corticosteroids (prednisone $>5$ $\mathrm{mg}$ /day), methotrexate, or sulfasalazine; and, for premenopausal women, pregnancy, considering pregnancy, or not using a reliable form of birth control. The study protocol was approved by the research ethics boards at McMaster University Medical Centre and St Joseph's Hospital, Hamilton, Ontario, Canada. Informed written consent was obtained from all patients.

\section{RANDOMISATION}

Consenting patients were randomly allocated in equal numbers to receive doxycycline 100 mg twice daily or an identical appearing placebo for 90 days. The study pharmacist filled the prescriptions by computer generated random assignment in blocks of six, and had sole access to the randomisation code. Throughout the study, patients, treating doctors, and laboratory personnel were unaware of treatment status, and patients and treating doctors were unaware of antibody status. After completion of the study in July 1998, patients allocated to placebo were offered one to three months of open label doxycycline. Seven patients crossed over 3-12 months after study enrolment. To avoid bias introduced by this cross over, no further patient data were accepted from either study arm after the study closing date.

BASELINE HISTORY AND RHEUMATOLOGICAL EXAMINATIONS

From the initial clinical encounter and the patient's medical record, a case report form was filled out giving details of age, sex, date of joint symptom onset, joints affected, and history of overseas travel before onset of joint symptoms, diarrhoea, pharyngitis, psoriasis, aphthous ulcers, conjunctivitis or iritis, urethritis, cervicitis, known chlamydial infection in the patient or partner, and other sexually transmitted diseases in the patient or partner.

During the initial rheumatological consultation an assessment was made of the affected joints and total joint count; enthesopathy; rashes; eye involvement; and skin involvement. The rheumatologist assessed pain, functional impairment, and overall arthritis activity using a $10 \mathrm{~cm}$ visual analogue scale (VAS, $0=$ best, $10=$ worst). Repeat examinations were performed at three, six, and 12 months, and consisted of joint count and VAS estimates of pain, functional impairment, and arthritis activity.
HEALTH RELATED QUALITY OF LIFE QUESTIONNAIRE

We measured health related quality of life using the second version of the Arthritis Impact Measurement Scales (AIMS2). ${ }^{24}$ The original AIMS questionnaire was designed specifically for patients with arthritis, and has demonstrated validity against clinical observation and other questionnaires, as well as showing responsiveness to change in clinical trials of treatment. $^{25-27}$ In the AIMS2 questionnaire, additional domains were added to include 12 domains in total. It is self administered and takes 20-25 minutes to complete.

AIMS2 questionnaires were completed before starting treatment, monthly while receiving treatment (one, two, and three months), and at six and 12 months or at the end of the study. Patients who discontinued treatment were encouraged to complete questionnaires at the prespecified times.

\section{STATISTICAL ANALYSIS}

Data from case report forms, AIMS2 questionnaires, and laboratory testing were analysed with BMDP (Biomedical Data Programs Inc, 1990, Los Angeles, CA) and SPSS software (SPSS version 10 for Windows, SPSS Inc, Chicago, IL). The mean or median of clinical and laboratory factors was examined for balance between treatment groups. The AIMS2 pain scale consists of five questions (each scored 1-5), which were summed to give a single pain score of between 5 (worst) and 25 (best). For composite functional score, four separate AIMS2 scales (mobility, walking and bending, self care, and household tasks) were summed and responses inverted as appropriate to ensure consistency ( 1 = worst, 4 or $5=$ best $)$, for a score of 18 (worst) to 90 (best). All 12 AIMS scales are presented for descriptive purposes.

The primary study end points were three month AIMS2 pain and functional change scores. We performed unpaired $t$ tests of change scores for patients with complete baseline and three month AIMS2 questionnaires. In nine patients, two month questionnaires were used in the absence of three month questionnaires. An analysis of variance (ANOVA) model including block randomisation assignment was performed to examine homogeneity across strata before pooling.

The secondary study end points were three month changes in rheumatologist assessed joint count, pain, functional status, and overall arthritis activity, and patient assessed pain and functional status at 6-12 months. We performed an a priori subgroup analysis for treatment effect in patients with a history or serological evidence of previous chlamydial or streptococcal infection by including interaction variables in an ANOVA model.

For case-control comparisons of Chlamydia trachomatis and Streptococcus pyogenes seroprevalence, patients were compared with age and sex matched outpatient controls by logistic regression, with patient status as the dependent variable (SPSS). 
Table 1 Baseline patient characteristics by treatment group. Results are shown as No (\%) unless otherwise stated

\begin{tabular}{|c|c|c|c|}
\hline & Doxycycline & Placebo & $p^{*}$ \\
\hline Total number & 30 & 30 & \\
\hline Evaluable at 3 months & $16 / 30(53)$ & $21 / 30(70)$ & 0.29 \\
\hline Age (median (quartiles)) & $45.0(32.8,51.3)$ & $43.5(39.0,50.3)$ & 0.48 \\
\hline Femal sex & $18 / 30(60)$ & $17 / 30(57)$ & 0.80 \\
\hline Duration (median (quartiles)) & $36(12,63)$ & $24(6,69)$ & 0.20 \\
\hline Gastrointestinal reactive & $0 / 30(0)$ & $3 / 30(10)$ & 0.24 \\
\hline Genitourinary reactive & $8 / 30(27)$ & $10 / 30(33)$ & 0.79 \\
\hline Undifferentiated seronegative arthritis & $15 / 30(50)$ & $12 / 30(40.0)$ & 0.60 \\
\hline Psoriatic arthritis & $5 / 30(17)$ & $3 / 30(10)$ & 0.71 \\
\hline Sacroiliitis only & $1 / 30(3)$ & $1 / 30(3)$ & 1.00 \\
\hline No acute arthritis & $1 / 30(3)$ & $1 / 30(3)$ & 1.00 \\
\hline History of travel & $0 / 30(0)$ & $1 / 30(3)$ & 1.00 \\
\hline History of diarrhoea & $1 / 30(3)$ & $6 / 30(20)$ & 0.10 \\
\hline History of eye symptoms & $6 / 30(20)$ & $11 / 30(37)$ & 0.25 \\
\hline History of urethritis/cervicitis & $1 / 30(3)$ & $1 / 30(3)$ & 1.00 \\
\hline History of chlamydia infection & $2 / 30(7)$ & $3 / 30(10)$ & 1.00 \\
\hline History of chlamydia in partner & $0 / 30(0)$ & $0 / 30(0)$ & 1.00 \\
\hline History of streptococcal infection & $1 / 30(3)$ & $1 / 30(3)$ & 1.00 \\
\hline$C$ trachomatis IgG or IgA $(\mathrm{n}=42)$ & $7 / 21(33)$ & $10 / 21(48)$ & 0.53 \\
\hline C. trachomatis by serology or history & $7 / 30(23)$ & $12 / 30(40)$ & 0.22 \\
\hline$S$ pyogenes serology $(\mathrm{n}=45)$ & $3 / 23(13)$ & $8 / 22(36)$ & 0.14 \\
\hline Joint count (median (quartiles, $\mathrm{n}=53$ )) & $3(2,5)$ & $5(2,8)$ & 0.14 \\
\hline VAS $†$ pain (mean $(S D), n=36)$ & $4.3(2.4)$ & $3.6(3.1)$ & 0.49 \\
\hline VAS function (mean (SD), $n=36$ ) & $4.0(2.4)$ & $2.2(2.3)$ & 0.27 \\
\hline VAS arthritis activity (mean (SD) $n=36$ ) & $4.5(2.8)$ & $3.6(2.9)$ & 0.33 \\
\hline
\end{tabular}

${ }^{\star} \chi^{2}$ (with Yates's correction) or Fisher exact test used for comparison of proportions; $t$ test for comparison of means; Mann-Whitney U test for comparison of ranks.

tVAS = visual analogue scale $(0-10 \mathrm{~cm}, 0=$ best, $10=$ worst $)$.

All analyses were performed on an intention to treat basis. For primary end points, $p<0.05$ was considered significant. The results of secondary analyses were judged significant for $\mathrm{p}<0.05$ only if the primary analyses were also significant.

\section{LABORATORY METHODS}

To detect active genitourinary $C$ trachomatis infection, a first void urine was tested for $C$ trachomatis nucleic acid amplification (LCx, Abbott, Chicago, IL). Serum IgG and IgA antibodies were measured by a recombinant polypeptide enzyme immunoassay specific for $C$ trachomatis (SeroCT, Savyon Diagnostics, Kiryat Minrat, Israel). Serum was diluted $1: 105$, incubated for 30 minutes at $37^{\circ} \mathrm{C}$, conjugated for 30 minutes with anti-IgG or anti-IgA antibody, and read in a spectrophotometer at $450 \mathrm{~nm}$. A cut off level was determined as twice the mean of three negative control sera, and a cut off index of 1.1 or greater was considered positive, as recommended by the manufacturer. Serum (diluted $1: 100)$ was also tested for serological evidence of previous Streptococcus pyogenes infection by a slide agglutination assay (Streptozyme, Wampole Laboratories, Cranbury, NJ) that measures antibodies to five streptococcal exoantigens (streptolysin $\mathrm{O}$, hyaluronidase, DNase B, NADase, and streptokinase).

\section{Results}

PATIENT DESCRIPTION

Sixty patients were enrolled between January 1997 and June 1998. Patients had a median age of 44 years (range 18-65), median of 24 months of arthritis symptoms (4-432 months), and $35 / 60(58 \%)$ were female (table 1). Back involvement alone was present in two $(3 \%)$ patients, peripheral joints alone in $50(83 \%)$, and eight (13\%) were affected in both. Extra-articular manifestations were uncommon, with a history of urethritis or cervicitis in two (3\%) patients, diarrhoea in the month preceding onset of arthritis in seven (12\%) patients, and a psoriatic skin rash in eight $(13 \%)$. Patients were concurrently taking nonsteroidal anti-inflammatory drugs, but none were taking disease modifying agents at study enrolment. Four patients started disease modifying drugs during the study, including sulfasalazine, methotrexate, or prednisone.

Six $(10 \%)$ patients had previous chlamydia or other sexually transmitted disease, and none reported partners with previous sexually transmitted diseases. One patient of $59(2 \%)$ tested had a positive first void urine for $C$ trachomatis nucleic acid. Serological evidence of previous $C$ trachomatis infection (IgG or IgA positive) was present in $17 / 42(40 \%)$ tested, compared with $7 / 42(17 \%)$ age and sex matched controls (odds ratio (OR) 3.3, 95\% confidence interval (CI) 1.2 to $9.2, \mathrm{p}=0.02)$. Eleven of $45(24 \%)$ tested had evidence of recent streptococcal infection, compared with $8 / 45$ (18\%) controls (OR 1.5, 95\% CI 0.5 to $4.0, \mathrm{p}=0.47$ ). Serological testing for Lyme disease and for syphilis, in 39 and 36 patients respectively, and stool cultures in 35 patients, were all negative.

At study baseline, AIMS2 questionnaires were completed by 58 patients. Patients identified moderately severe pain scores (mean 11.3 of 25) and mildly impaired composite functional scores (mean 73.2 of 90). Of the four scales in the composite functional score, the greatest impairment was seen in the walking and bending scale (mean 14.6 of 25). Moderate difficulty was identified in the hand and finger (9.6 of 25), arm (9.0 of 25), and family and friends (7.1 of 20$)$ scales.

Thirty patients each were allocated to receive doxycycline or placebo. Groups were well balanced for potential prognostic factors (see table 1). Age, sex, years of arthritis, extraarticular manifestations, joint counts, and exposure to $C$ trachomatis or Streptococcus pyogenes were similar in both groups. AIMS2 questionnaire scores (table 2) were similar for the two groups, except for arm function, which was better in the doxycycline than the placebo group.

PATIENT FOLLOW UP, COMPLIANCE, AND SIDE EFFECTS

Thirty seven patients (62\%) completed the study, including 16 assigned to active treatment and 21 assigned to placebo. Six month questionnaires were available for $15(25 \%)$ patients, and 12 month questionnaires for six $(10 \%)$ patients. Three month rheumatology case report forms were available for $25(42 \%)$ patients; six month forms for $12(20 \%)$ patients; and 12 month forms for eight (13\%) patients. Of the 23 patients lost to follow up, two withdrew consent after randomisation without starting treatment; two did not fill out baseline or subsequent questionnaires; four started disease remitting agents and did not fill out follow up questionnaires; two stopped active treatment owing to side effects; five did 
Table 2 Baseline mean scores from the Arthritis Impact Measurement Scales version 2 (AIMS2) instrument by treatment group $(n=58)$

\begin{tabular}{lccccc}
\hline & \multicolumn{5}{c}{ Mean scores } \\
\cline { 5 - 6 } AIMS2 Scale & Possible scores* & Patient scores & Doxycycline & Placebo & pt \\
\hline 1 Pain & $5-25$ & $5-22$ & 10.9 & 11.7 & 0.46 \\
2-5 Functional score $\neq$ & $18-90$ & $41-90$ & 73.4 & 73.1 & 0.92 \\
& & & & & \\
2 Mobility & $5-25$ & $9-25$ & 21.9 & 21.3 & 0.58 \\
3 Walking and bending & $5-25$ & $9-25$ & 14.5 & 14.8 & 0.77 \\
4 Self care & $4-20$ & $10-20$ & 18.7 & 18.9 & 0.73 \\
5 Household tasks & $4-20$ & $11-20$ & 18.3 & 18.1 & 0.74 \\
& & & & & \\
6 Hand and finger & $5-25$ & $5-19$ & 10.4 & 8.8 & 0.13 \\
7 Arm & $5-25$ & $5-21$ & 10.3 & 7.7 & 0.01 \\
8 Mood & $5-25$ & $19-24$ & 18.7 & 18.3 & 0.67 \\
10 Famsion & $5-25$ & $8-23$ & 15.6 & 14.4 & 0.19 \\
11 Social activity & $4-20$ & $5-19$ & 7.0 & 7.2 & 0.76 \\
12 Work & $5-25$ & $8-23$ & 15.3 & 16.5 & 0.17 \\
& $4-20$ & $6-20$ & 14.2 & 14.3 & 0.92 \\
\hline
\end{tabular}

${ }^{\star}$ Lower numbers indicate worse score.

†Comparison of mean scores by unpaired $t$ test (SPSS).

$\ddagger$ Composite functional score consists of sum of mobility, walking and bending, self care, and household tasks AIMS2 scales.

not attend for further rheumatology follow up; and eight attended rheumatology follow up but did not submit questionnaires. There were no statistically significant differences in baseline characteristics between patients lost to follow up and those completing the study. Side effects were reported infrequently, and included photosensitivity in two patients, oesophagitis in one patient, and gastric upset in one other. One patient experienced a flare of finger and wrist pain and swelling after starting doxycycline, which improved when it was stopped and immediately recurred after rechallenge. One patient developed hepatitis during open label doxycycline treatment after completion of the study. Liver enzymes improved after stopping treatment, but he declined a liver biopsy and the cause of his hepatitis remains unclear.

PATIENT OUTCOMES

Table 3 lists patient outcomes at three months $(\mathrm{n}=37)$. AIMS2 pain scores $(5=$ worst, $25=$ best) increased from study enrolment to three months in both groups, from 10.8 to 13.5 (doxycycline), and from 12.7 to 13.8 (placebo), for a mean treatment difference in pain change scores of 1.5 points $(95 \% \mathrm{CI}-1.2$ to $4.2, \mathrm{p}=0.25$ ). Composite functional scores (the sum of the four AIMS2 scales of mobility, walking and bending, self care, and household tasks; $18=$ worst, $90=$ best) increased in both groups, from 71.9 to 75.1 (doxycycline), and from 73.0 to 75.4 (placebo), for a mean difference in change scores of $0.8(95 \% \mathrm{CI}-5.6$ to $7.1, \mathrm{p}=0.81)$. The four individual function scales were similar between active treatment and placebo, and no statistically significant differences were detected for the remaining seven AIMS2 scales (hand and finger, arm, mood, tension, family and friends, social activity, and work scales).

At the three month rheumatology assessment $(n=25)$, joint counts increased in both study groups (mean difference 1.6, 95\% CI -1.6 to $4.7, \mathrm{p}=0.32$ ). Rheumatologist assessed pain (visual analogue change score mean difference $-0.6 \mathrm{~cm}$ ), functional score (mean difference $-0.9 \mathrm{~cm}$ ), and overall arthritis activity (mean difference $-0.6 \mathrm{~cm}$ ) were similar for doxycycline and placebo groups (table 3 ).

At 6-12 months, AIMS2 pain scores were 15.2 (doxycycline) and 14.5 (placebo), with mean change scores of $3.5 v 2.2$ (mean difference $1.3,95 \% \mathrm{CI}-5.6$ to $8.2, \mathrm{p}=0.69)$. Composite functional scores were 70.8 (doxycycline) and 73.7 (placebo), with mean change scores of $-2.0 v 0.2$ (mean difference -2.2 , $95 \% \mathrm{CI}-14.2$ to $9.8, \mathrm{p}=0.70)$. These analyses were based on 16 patients. A repeated measures ANOVA based on 15 patients and three to five available time periods found no treatment effect on pain $(p=0.48)$ and composite functional score $(\mathrm{p}=0.71)$.

SUBGROUP ANALYSES FOR TREATMENT EFFICACY Of the 37 patients evaluable at three months, 11 patients were $C$ trachomatis serology status

Table 3 Three month treatment effect of doxycycline compared with placebo for chronic seronegative or reactive arthritis

\begin{tabular}{|c|c|c|c|c|c|c|c|c|}
\hline & \multicolumn{3}{|c|}{ Doxycycline $(n=16)$} & \multicolumn{3}{|c|}{ Placebo $(n=21)$} & \multicolumn{2}{|l|}{ Comparison } \\
\hline & $0 \mathrm{~m}$ & $3 m$ & Change & $0 \mathrm{~m}$ & $3 \mathrm{~m}$ & Change & $\begin{array}{l}\text { Mean difference } \\
(95 \% \mathrm{CI})\end{array}$ & $p^{\star}$ \\
\hline AIMS2† pain & 10.8 & 13.5 & 2.7 & 12.7 & 13.8 & 1.1 & $1.5(-1.2$ to 4.2$)$ & 0.25 \\
\hline AIMS2 function $\ddagger$ (4 scales) & 71.9 & 75.1 & 3.2 & 73.0 & 75.4 & 2.4 & $0.8(-5.6$ to 7.1$)$ & 0.81 \\
\hline AIMS2 mobility & 21.5 & 22.1 & 0.6 & 21.1 & 21.7 & 0.6 & $0.0(-3.2$ to 3.1$)$ & 0.97 \\
\hline AIMS 2 walking and bending & 13.2 & 16.3 & 3.2 & 15.1 & 16.6 & 1.6 & $1.6(-0.9$ to 4.2$)$ & 0.21 \\
\hline AIMS2 self care & 18.9 & 19.4 & 0.6 & 19.1 & 19.2 & 0.2 & $0.4(-1.1$ to 1.8$)$ & 0.61 \\
\hline AIMS2 household tasks & 18.4 & 17.3 & -1.1 & 17.9 & 17.9 & 0.0 & $-1.2(-3.0$ to 0.6$)$ & 0.19 \\
\hline AIMS2 hand and finger & 11.1 & 10.5 & -0.6 & 8.8 & 8.2 & -0.6 & $0.0(-2.3$ to 2.2$)$ & 0.97 \\
\hline AIMS2 arm & 10.9 & 9.1 & -1.9 & 7.4 & 6.8 & -0.6 & $-1.3(-3.0$ to 0.5$)$ & 0.15 \\
\hline AIMS2 mood & 18.5 & 19.2 & 0.7 & 19.0 & 20.0 & 1.0 & $-0.3(-2.0$ to 1.4$)$ & 0.72 \\
\hline AIMS2 tension & 14.8 & 15.3 & 0.5 & 16.2 & 17.6 & 1.4 & $-0.9(-3.0$ to 1.2$)$ & 0.38 \\
\hline AIMS2 family and friends & 6.8 & 7.4 & 0.6 & 7.2 & 8.5 & 1.3 & $-0.7(-2.6$ to 1.1$)$ & 0.41 \\
\hline AIMS2 social activity & 15.6 & 15.5 & -0.1 & 16.5 & 16.5 & 0.0 & $-0.1(-2.7$ to 2.6$)$ & 0.96 \\
\hline AIMS2 work & 12.2 & 12.2 & 0.0 & 14.9 & 17.4 & 2.5 & $-2.5(-5.4$ to 0.5$)$ & 0.10 \\
\hline Joint count & 4.0 & 5.6 & 1.6 & 5.4 & 5.5 & 0.1 & $1.6(-1.6$ to 4.7$)$ & 0.32 \\
\hline Pain score (VAS $\S)$ & 4.3 & 4.6 & 0.3 & 3.6 & 4.5 & 0.9 & $-0.6(-3.1$ to 1.9$)$ & 0.46 \\
\hline Functional score (VAS) & 4.0 & 3.7 & -0.3 & 2.2 & 2.8 & 0.7 & $-0.9(-2.5$ to 0.7$)$ & 0.15 \\
\hline Arthritis activity (VAS) & 4.5 & 4.7 & 0.1 & 3.6 & 4.4 & 0.8 & $-0.6(-3.1,1.9)$ & 0.48 \\
\hline
\end{tabular}

*Change score means compared by unpaired $t$ test (SPSS).

†AIMS2 = Arthritis Impact Measurement Scales version 2 self assessed questionnaire $(n=37)$. Lower numbers indicate a worse score. AIMS2 pain and composite functional scores constituted the primary study end points.

¥Composite functional score consists of sum of mobility, walking and bending, self care, and household tasks AIMS 2 scales.

\VAS = visual analogue scale $(0=$ best, $10=$ worst $)$ assessed by rheumatologist $(n=25)$. 
Table 4 Treatment effect of doxycycline compared with placebo for chronic seronegative or reactive arthritis on pain and functional change scores (AIMS2) by chlamydia and streptococcus status

\begin{tabular}{|c|c|c|c|c|c|c|c|}
\hline & \multirow[b]{2}{*}{$n$} & \multirow[b]{2}{*}{ AIMS2 scale* } & \multicolumn{3}{|c|}{ Change Scores } & \multirow[b]{2}{*}{$F$ test } & \multirow[b]{2}{*}{$p t$} \\
\hline & & & Doxycycline & Placebo & $\begin{array}{l}\text { Mean difference ( } 95 \% \\
\text { CI) }\end{array}$ & & \\
\hline CT $\ddagger$ positive & 11 & Pain (3 m) & 3.3 & 1.3 & $2.1(-2.7$ to 6.8$)$ & & 0.35 \\
\hline CT unknown & 26 & Pain (3 m) & 2.5 & 1.1 & $1.5(-2.1$ to 5.1$)$ & & 0.41 \\
\hline CT interaction $\rrbracket$ & 37 & Pain (3 m) & & & & 0.02 & 0.90 \\
\hline CT positive & 11 & Functional $(3 \mathrm{~m})$ & 2.3 & 6.6 & $-4.3(-19.9$ to 11.1$)$ & & 0.55 \\
\hline CT unknown & 26 & Functional ( $3 \mathrm{~m})$ & 3.4 & -0.2 & $3.6(-3.8$ to 10.9$)$ & & 0.33 \\
\hline CT interaction & 37 & Functional (3 m) & & & & 0.13 & 0.72 \\
\hline Strep ${ }^{\star \star}$ positive & 6 & Pain (3 m) & 3.0 & 0.2 & $2.8(-12.6$ to 18.2$)$ & & 0.64 \\
\hline Strep unknown & 31 & Pain (3 m) & 2.7 & 1.4 & $1.2(-1.7$ to 4.1$)$ & & 0.39 \\
\hline Strep interaction & 37 & Pain $(3 \mathrm{~m})$ & & & & 0.11 & 0.74 \\
\hline Strep positive & 6 & Functional (3 m) & 10.0 & -0.4 & $10.4(-12.3$ to 33.1$)$ & & 0.27 \\
\hline Strep unknown & 31 & Functional (3 m) & 2.7 & 3.3 & $-0.6(-7.8$ to 6.7$)$ & & 0.87 \\
\hline Strep interaction & 37 & Functional (3 m) & & & & 0.98 & 0.33 \\
\hline
\end{tabular}

^AIMS2 = Arthritis Impact Measurement Scales version 2 questionnaire $(\mathrm{n}=37)$.

†Change score mean difference and $95 \%$ confidence interval from unpaired $t$ test.

$\ddagger \mathrm{CT}=$ Chlamydia trachomatis positive by serology (IgA or IgA) or clinical history.

SInteraction term analysed in a full factorial ANCOVA model (SPSS).

TComposite functional score consists of sum of mobility, walking and bending, self care, and household tasks AIMS2 scales.

${ }^{\star} \star$ Strep $=$ Streptococcus pyogenes antibody positive by slide agglutination.

positive and 26 patients were serology negative or unknown. Treatment effects were examined by pain and functional status change scores by chlamydia status (table 4). In addition, the interaction of serological status and treatment was examined in an ANCOVA design. For pain at three months, pain scores improved 2.1 points (95\% CI -2.7 to 6.8 ) in the chlamydia positive group, compared with 1.5 points $(95 \%$ CI -2.1 to 5.1 ) in the chlamydia negative group. The chlamydia-by-treatment regression interaction term was non-significant $(\mathrm{p}=0.90)$. For functional status there was a decline of 4.3 points $(95 \% \mathrm{CI}-19.9$ to 11.1$)$ in the chlamydia positive group, compared with an improvement of $3.6(95 \% \mathrm{CI}-3.8$ to 10.9$)$ in the chlamydia negative group. The chlamydia-bytreatment interaction terms were not significant $(\mathrm{p}=0.72)$.

Of the 37 patients evaluable at three months, six patients were streptococcal status positive and for 31 patients streptococcal status was negative or unknown (table 4). Pain change scores at three months improved by 2.8 points (95\% CI -12.6 to 18.2$)$ and 1.2 points $(95 \%$ CI -1.7 to 4.1 ) in the streptococcal status positive and negative groups, respectively. Composite functional change scores improved 10.4 points (95\% CI -12.3 to 33.1$)$ and -0.6 points $(95 \% \mathrm{CI}-7.8$ to 6.7$)$ in the streptococcal status positive and negative groups, respectively. The streptococcus-by-treatment interaction terms were not significant $(p=0.74$ for pain change scores and $p=0.33$ for functional change scores).

A treatment effect could not be shown in any other subgroup examined. For pain or functional change scores, there were no significant differences in outcome comparing men $v$ women $(\mathrm{p}=0.74$ for pain and $\mathrm{p}=0.56$ for functional score, respectively); age $<40$ years $v 40$ or older $(p=0.60$ and $p=0.50)$; duration of symptoms $<1$ year $v \geqslant 1$ year $(p=0.64$ and $\mathrm{p}=0.94)$; central involvement $v$ no central involvement $(\mathrm{p}=0.69$ and $\mathrm{p}=0.11)$; or presence $v$ absence of psoriatic lesions $(\mathrm{p}=0.69$ and $\mathrm{p}=0.87)$.

\section{Discussion}

Among patients with chronic reactive or undifferentiated seronegative arthritis, long term doxycycline did not improve patient or rheumatologist assessed pain, functional status, or disease activity at three, six, or 12 months. For the overall study, relatively narrow confidence intervals for pain and composite function change scores virtually rule out a clinically important improvement. Similarly, no benefit was seen in clinically important subgroups, such as those with previous chlamydial exposure, but the confidence intervals were much wider because the numbers of patients in the individual subgroups were small.

Exposure to chlamydia or streptococcal infections was common. Chlamydial seroprevalence was higher in patients than among age and sex matched controls, suggesting that some $60 \%$ of arthritis among those with positive serology could be attributed to $C$ trachomatis. In contrast, no association was found between arthritis symptoms and streptococcal serology.

The role of antibiotics for reactive or seronegative arthritis remains unclear. In a retrospective study, the incidence of reactive arthritis after non-gonococcal urethritis was lower with tetracycline based treatment than with penicillin based treatment or no treatment $(10 \% v 37 \%) .^{28}$ Thus timely treatment for presumed chlamydial infection may prevent subsequent reactive arthritis.

However, once arthritis has developed, antibiotics may not improve symptoms. Short courses of antibiotics for acute reactive arthritis were not effective in two randomised controlled trials. Five days' treatment with lincomycin for urethritis associated reactive arthritis, ${ }^{21}$ and 10-14 days of pathogen-specific treatment after gastrointestinal or genitourinary infection were all ineffective in decreasing symptoms, joint count, or inflammatory markers. $^{22}$

In two non-randomised studies, longer courses of antibiotics seemed to be more effective. Penicillin or minocycline for three 
months, ${ }^{29}$ or doxycycline for 3-4 months, ${ }^{30}$ improved pain, stiffness, functioning, inflammation, and clinical assessment of disease activity and prevented relapse during two years of follow up.

Four published randomised trials of long term antibiotic treatment for acute reactive or seronegative arthritis were identified, with total enrolment of 293 patients. ${ }^{23}{ }^{31-33}$ All these studies showed no overall improvement in symptoms, inflammatory markers, joint count, or time to improvement. Three of these studies used ciprofloxacin, a fluoroquinolone antibiotic with excellent activity for gastrointestinal pathogens and some activity against $C$ trachomatis, for three months ${ }^{31}{ }^{32}$ or 12 months. ${ }^{33}$ The fourth study used lymecycline, ${ }^{23}$ a tetracycline antibiotic with excellent $C$ trachomatis activity. In the latter study, 40 patients with acute (within two months of onset) reactive arthritis received lymecycline or placebo. In a subgroup analysis of 21 patients with chlamydia associated arthritis, but not in the overall study group, a statistically significant and clinically important difference in improvement was seen. Specifically, patients with serological evidence of chlamydia infection improved after a median of 15.0 weeks' treatment with lymecycline $v$ 39.5 weeks on placebo $(p=0.02)$. However, the optimal method and relevance of diagnosing chlamydial exposure by serology remain uncertain, and the findings of a subgroup analysis require independent confirmation.

In the largest of the four studies, ${ }^{32}$ three months' treatment with ciprofloxacin was ineffective both in the entire study group, and in subgroups defined by the length of symptoms (less than three months $v$ longer), clinical presentation (reactive $v$ undifferentiated oligoarthritis), or aetiology (genitourinary $v$ gastrointestinal). Patients with evidence of $C$ trachomatis infection (13/126) who were treated with ciprofloxacin showed an improvement in all indices except global health compared with placebo treated patients. However, only 10 patients were evaluable for the treatment end points, and a greater percentage of patients randomly allocated to the ciprofloxacin arm had symptoms for less than three months. Although the authors did not present a statistical analysis of the chlamydia infected subgroup, it is likely that neither unadjusted or baseline-covariate adjusted differences were statistically significant. Nevertheless, the results suggest that a treatment effect in chlamydia associated arthritis cannot be ruled out.

In this study we extend the observations of these previous studies to a different antibiotic regimen with improved antichlamydial activity (doxycycline), and to patients who had chronic symptoms (median 24 months) and a wider spectrum of disease than in previous studies (spondyloarthropathy, psoriatic arthritis, and various seronegative oligoarthritides and polyarthritides).

Two important research questions remain. Firstly, a beneficial treatment effect early in the course of Chlamydia trachomatis associated reactive arthritis remains plausible. We did not study disease of acute onset, and had only 11 patients (with a median of 48 months of symptoms) with laboratory evidence of previous $C$ trachomatis exposure who completed the three month questionnaire. In an animal model of yersinia induced arthritis, antibiotics were effective if started within three days of infection, but not if delayed until the onset of arthritis. ${ }^{34}$ Studies targeting acute onset $C$ trachomatis associated arthritis are needed.

Secondly, we had few patients who returned questionnaires six months or longer after enrolment, and cannot rule out a possible long term antibiotic effect. By analogy with rheumatic heart disease, it is possible that years of antibiotic prophylaxis would prevent future exacerbations, but current disease flares may not be amenable to antibiotics.

The lack of treatment effect does not necessarily contradict the hypothesis of persistent antigenic stimulus by occult infection, because bacterial persistence in a form unresponsive to antibiotic treatment has been reported. ${ }^{35} 36$ Nevertheless, we conclude that doxycycline is not an effective treatment for chronic reactive or undifferentiated seronegative arthritis.

Special thanks to Norma Marchetti for random allocation of patients, drug preparation, and dispensing, to Santina Castripatients, drug preparation, and dispensing, to Santina Castriwith laboratory assays, and to Dr Bella Ohana and Savyon with laboratory assays, and to Dr Bella Ohana and Savyon
Diagnostics (Israel) for technical advice and provision of Diagnostics (Israel) for technical ad
Chlamydia trachomatis diagnostic kits.

This work was supported by the Physicians of Ontario through a grant from the PSI Foundation, and by the Father Sean O'Sullivan Research Centre at St. Joseph's Hospital, Hamilton, ON, Canada.

1 Kean WF, MacPherson DW. Reiter's syndrome. In: Bellamy $\mathrm{N}$, ed. Prognosis in the rheumatic diseases. Dordrecht: Kluwer Academic Publishers, 1991:167-92.

2 Sieper J, Braun J, Kingsley GH. Report on the Fourth International Workshop on Reactive Arthritis. Arthritis Rheum 2000;43:720-34.

3 Keat A. Reiter's syndrome and reactive arthritis in perspective. N Engl J Med 1983;309:1606-15.

4 Thomson GT, DeRubeis DA, Hodge MA, Rajanayagam C, Inman RD. Post-salmonella reactive arthritis: late clinical sequelae in a point source cohort. Am J Med 1995;98:1321 .

5 Keat AC, Thomas BJ, Taylor-Robinson D, Pegrum GD, Kaini RN, Scott JT. Evidence of Chlamydia trachomatis
Mat infection in sexually acquired reactive arthritis. Ann infection in sexually acquire

Rheum Dis 1980;39:431-7.
6 Rich E, Hook EW III, Alarcon GS, Moreland LW. Reactive arthritis in patients attending an urban sexually transmitted diseases clinic. Arthritis Rheum 1996;39:1172-7.

7 Csonka GW. The course of Reiter's syndrome. BMJ 1958;i: 1088-90.

8 Hughes R, Keat A. Reactive arthritis: the role of bacterial antigens in inflammatory arthritis. Baillieres Clin Rheumatol 1992;6:285-308

9 Deighton C. Beta haemolytic streptococci and reactive arthritis in adults. Ann Rheum Dis 1993;52:475-82.

10 Braun J, Laitko S, Treharne J, Eggens U, Wu P, Distler A, et al. Chlamydia pneumoniae-a new causative agent of reactive arthritis and undifferentiated oligoarthritis. Ann Rheum Dis 1994;53:100-5.

11 Weyend CM, Goronzy JJ. Clinically silent infections in patients with oligoarthritis: results of a prospective study. patients with oligoarthritis: result

12 Sieper J, Braun J, Brandt J, Miksits K, Heesemann J, Laitko $\mathrm{S}$, et al. Pathogenetic role of chlamydia, yersinia and borrelia in undifferentiated oligoarthritis. J Rheumatol 1992;19: $1236-42$.

13 Inman RD, Whittum-Hudson JA, Schumacher HR, Hudson AP. Chlamydia and associated arthritis. Curr Opin Rheumatol 2000;12:254-62.

14 Keat A, Thomas B, Dixey J, Osborn M, Sonnex C, TaylorRobinson D. Chlamydia trachomatis and reactive arthritis: the missing link. Lancet 1987;i:72-4.

15 Rahman MU, Hudson AP, Schumacher HR. Chlamydia and Reiter's syndrome (reactive arthritis). Rheum Dis Clin North Am 1992;18:67-79.

16 Csonka GW. Recurrent attacks in Reiter's disease. Arthritis Rheum 1960;3:164-9.

17 Schumacher HR Jr, Magge S, Cherian PV, Sleckman J, Rothfuss S, Clayburne G, et al. Light and electron microscopic studies on the synovial membrane in Reiter's syndrome. Immunocytochemical identification of chlamydial antigen in patients with early disease. Arthritis Rheum 1988;31:937-46. 
18 Taylor-Robinson D, Gilroy CB, Thomas BJ, Keat ACS. Detection of Chlamydia trachomatis DNA in joints of
reactive arthritis patients by polymerase chain reaction. reactive arthritis patien
Lancet 1992;340:81-2.

19 Braun J, Tuszewski M, Eggens U, Mertz A, SchauerPetrowskaja C, Doring E, et al. Nested polymerase chain reaction strategy simultaneously targeting DNA sequences of multiple bacterial species in inflammatory joint diseases. I. Screening of synovial fluid samples of patients with spondyloarthropathies and other arthritides. J Rheumato 1997;24:1092-100.

20 Wilkinson NZ, Kingsley GH, Jones HW, Sieper J, Braun J, Ward ME. The detection of DNA from a range of bacterial species in the joints of patients with a variety of arthritides using a nested, broad-range polymerase chain reaction. Rheumatology (Oxford) 1999;38:260-6.

21 Whaley K, Downie WW, Dick WC, Nuki G, Schofield CB, Anderson J. Clinical trial of lincomycin hydrochloride in Anderson J. Clinical trial of lincomyci

22 Fryden A, Bengtsson A, Foberg U, Svenungsson B, Casto B, Karnell A, et al. Early antibiotic treatment of reactive B, Karnell A, et al. Early antibiotic treatment of reactive arthritis associated with enteric infections:
serological study. BMJ 1990;301:1299-302.

23 Lauhio A, Leirisalo-Repo M, Lahdevirta J, Saikku P, Repo $\mathrm{H}$. Double-blind, placebo-controlled study of three-month treatment with lymecycline in reactive arthritis, with special reference to chlamydia arthritis. Arthritis Rheum 1991;34: 6-14.

24 Meenan RF, Mason JH, Anderson JJ, Guccione AA, Kazis LE. AIMS2. The content and properties of a revised and expanded Arthritis Impact Measurement Scales Health Status Questionnaire. Arthritis Rheum 1992;35:1-10.

25 Meenan RF, Gertman PM, Mason JH. Measuring health status in arthritis. The arthritis impact measurement scales. Arthritis Rheum 1980;23:146-52.

26 Meenan RF, Gertman PM, Mason JH, Dunaif R. The arthritis impact measurement scales. Further investigations of a health status measure. Arthritis Rheum 1982;25:104853.
27 Anderson JJ, Firschein HE, Meenan RF. Sensitivity of a health status measure to short-term clinical changes in health status measure to short-term clinical
arthritis. Arthritis Rheum 1989;32:844-50.

28 Bardin T, Enel C, Cornelis F, Salski C, Jorgensen C, Ward $\mathrm{R}$, et al. Antibiotic treatment of venereal disease and Reiter's syndrome in a Greenland population. Arthritis Rheum 1992;35:190-4.

29 Panayi GS, Clark B. Minocycline in the treatment of patients with Reiter's syndrome. Clin Exp Rheumatol 1989;7:101-2

30 Pott HG, Wittenborg A, Junge-Hulsing G. Long-term antibiotic treatment in reactive arthritis. Lancet 1988;i:245-6.

31 Yli-Kerttula T, Luukkainen R, Yli-Kerttula U, Mottonen T, Hakola M, Korpela M, et al. Effect of a three month course of ciprofloxacin on the outcome of reactive arthritis. Ann Rheum Dis 2000;59:565-70.

32 Sieper J, Fendler C, Laitko S, Sorensen H, GripenbergLerche C, Hiepe F, et al. No benefit of long-term ciprofloxacin treatment in patients with reactive arthritis and undifferentiated oligoarthritis: a three-month, multicenter, double-blind, randomized, placebo-controlled center, double-blind, randomized, place

33 Wakefield D, McCluskey P, Verma M, Aziz K, Gatus B, Carr Wa Ciprofloxacin treatment does not influence course or $G$. Ciprofloxacin treatment does not influence course or
relapse rate of reactive arthritis and anterior uveitis. Arthritis Rheum 1999;42:1894-7.

34 Zhang Y, Gripenberg-Lerche C, Soderstrom KO, Toivanen A, Toivanen P. Antibiotic prophylaxis and treatment of reactive arthritis. Lessons from an animal model. Arthritis Rheum 1996;39:1238-43.

35 Nanagara R, Li F, Beutler A, Hudson A, Schumacher HR. Alteration of Chlamydia trachomatis biologic behavior in synovial membranes. Suppression of surface antigen production in reactive arthritis and Reiter's syndrome. Arthritis Rheum 1995;38:1410-17.

36 Gerard HC, Branigan PI, Schumacher HR, Hudson AP. Synovial Chlamydia trachomatis in patients with reactive arthritis/Reiter's syndrome are viable but show aberrant gene expression. J Rheumatol 1998;25:734-42. 